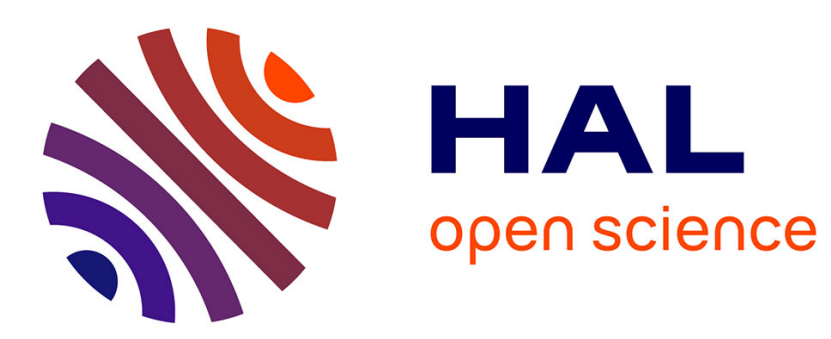

\title{
Fast segmentation of ultrasound images by incorporating spatial information into Rayleigh mixture model
}

\author{
Hui Bi, Hui Tang, Guanyu Yang, Baosheng Li, Huazhong Shu, Jean-Louis
} Dillenseger

\section{- To cite this version:}

Hui Bi, Hui Tang, Guanyu Yang, Baosheng Li, Huazhong Shu, et al.. Fast segmentation of ultrasound images by incorporating spatial information into Rayleigh mixture model. IET Image Processing, 2017, 11 (12), pp.1188-1196 10.1049/iet-ipr.2017.0166 . inserm-01635317

\section{HAL Id: inserm-01635317 https://www.hal.inserm.fr/inserm-01635317}

Submitted on 15 Nov 2017

HAL is a multi-disciplinary open access archive for the deposit and dissemination of scientific research documents, whether they are published or not. The documents may come from teaching and research institutions in France or abroad, or from public or private research centers.
L'archive ouverte pluridisciplinaire HAL, est destinée au dépôt et à la diffusion de documents scientifiques de niveau recherche, publiés ou non, émanant des établissements d'enseignement et de recherche français ou étrangers, des laboratoires publics ou privés. 


\title{
Fast Segmentation of Ultrasound Images by Incorporating Spatial Information into Rayleigh Mixture Model
}

\author{
Hui Bi ${ }^{1,2,3}$, Hui Tang ${ }^{1,2,3}$, Guanyu Yang ${ }^{1,2,3}$, Baosheng Li $^{4}$, Huazhong Shu ${ }^{1,2,3, *}$, Jean-Louis Dillenseger ${ }^{5,6,3}$ \\ ${ }^{1}$ Laboratory of Image Science and Technology, School of Computer Science and Engineering, \\ Southeast University, 210096 Nanjing China \\ ${ }^{2}$ Key Laboratory of Computer Network and Information Integration (Southeast University), Min- \\ istry of Education, 210096 Nanjing China \\ ${ }^{3}$ Centre de Recherche en Information Biomédicale sino-français, 210096 Nanjing China \\ ${ }^{4}$ Department of Radiation Oncology, Shandong Cancer Hospital, 250117 Jinan, China \\ ${ }^{5}$ INSERM U1099, 35000 Rennes, France \\ ${ }^{6}$ Laboratoire Traitement du Signal et de l'Image, Université de Rennes I, 35000 Rennes, France \\ *shu.list@seu.edu.cn
}

\begin{abstract}
As a particular case of the Finite Mixture Model (FMM), Rayleigh Mixture Model (RMM) is considered as a useful tool for medical ultrasound (US) image segmentation. However, conventional RMM relies on intensity distribution only and does not take any spatial information into account that leads to misclassification on boundaries and inhomogeneous regions. In this paper, we propose an improved RMM with Neighbour information (RMMN) to solve this problem by introducing neighbourhood information through a mean template. The incorporation of the spatial information made RMMN more robust to noise on the boundaries. The size of the window which incorporates neighbour information was resized adaptively according to the local gradient distribution. We evaluated our model on experiments on synthetic data and real US images used by High-Intensity Focused Ultrasound (HIFU) therapy. On this data, we demonstrated that that the proposed model outperforms several state-of-art methods in terms of both segmentation accuracy and computation time.
\end{abstract}

\section{Introduction}

Image segmentation is an active research topic in image analysis and computer vision. It simplifies the understanding of an image from thousands of pixels to a few regions of interests. Broadly, the segmentation methods can be divided into four categories: threshold-based, clustering, edge detection, and region growing methods [1].

There are several image segmentation techniques [2]. Traditional threshold-based methods such as Otsus depend on grey level histogram are widely used because of their simplicity and efficiency. However, they are generally not suitable for medical image since the grey level histogram is more complex. Clustering methods are commonly applied for medical image segmentation. Among them, $K$-means means algorithm is a commonly used unsupervised clustering method. The main disadvantage of the $K$-means algorithm is that it relies too much on the initial values. A bad initialization leads generally to a bad segmentation result.

Finite Mixture Model (FMM) is one of the most widely used clustering models for image seg- 
mentation. Mathematically, it can be considered as a linear combination of prior and conditional probabilities [3, 4]. Image segmentation and classification based on FMM has been reported in the literature $[5,6,7]$. A survey about the finite mixture model can be found in [8]. The key point of any FMM is the choice of the probability distribution which mainly depends on the front-end applications. Gaussian Mixture Model (GMM) is the most commonly used case of FMM by assuming that the intensity distribution follows a Gaussian distribution $[9,10,11]$. The choice of a Gaussian distribution is justified by the fact that it approximates the asymptotic behaviour of many probability distributions. The main advantage of GMM is that it is easy to implement and it has only a few parameters. In a GMM, the parameter learning can be easily achieved by using the maximum likelihood algorithm (ML) or the expectation-maximization (EM) algorithm $[12,13,14,15,16]$.

The segmentation of Ultrasound (US) image is more particular compared to the segmentation of other medical images modalities [17]. In US images, the information is mainly composed of speckle patterns. Speckles come from the interference of the US signal scattered by the tissues micro in-homogeneities (tissues cells, capillaries, blood cells, and so on). Because the spatial distribution of the micro in-homogeneities is specific to each tissue, the speckle pattern is also specific to each tissue. The assumption can be stated that some statistics on the speckle distribution can be used to characterize the observed tissues [18]. Jakeman [19] and Insana et al. [20] made the assumption that the speckle intensities in US data follow a Rician distribution. Dutt et al. [21] and Prager et al. [22] claimed that the distribution is more likely a $K$ distribution. Shankar [23] considered that the data follows a Nakagami distribution. But more generally, when a large amount of scatterers is locally distributed inside a small area, it has been demonstrated that the statistics of the envelop of the received signal follow a Rayleigh distribution [18, 24]. For this reason, some authors proposed to adapt the FMM segmentation framework for the US data analysis by estimating a Rayleigh Mixture Model (RMM) [25, 26]. When it is applied to US images, RMM seems to be a more flexible and powerful statistical model for data classification. For example, Seabra et al. [25] made use of RMM as a feature for intravascular US image classification. In their texture classification framework, RMM seems to provide one of the most pertinent texture features to accurately describe different plaque types and so to significantly improve the characterization performance. Pereyra et al. [26] found that $\alpha$-Rayleigh distributions were able to represent the statistics of ultrasound images for skin lesions characterization.

However, as any other models based only on the image intensities, the performance of the RMM is sensitive to noise and image level contrast. In order to overcome this problem, various methods have been proposed to incorporate neighbourhood information into FMM, focused on prior or conditional probability. Mixture models with Markov Random Field (MRF) have been employed to impose spatial constraints between neighbouring pixels in GMM [27, 28] and more recently in RMM [26]. In this class of methods, the MRF models the joint distribution of the priors between each pixel labels. However, the use of MRF in the model estimation has two main drawbacks. First it induces a growth of the number of parameters and second it has to be estimated at each iteration of the EM algorithm. These drawbacks lead to an increase of the computational complexity. With lower complexity, Tang et al. [29] modified the classical FMM intensity-only distributions estimation by incorporating spatial information to GMM through a mean template. More recently, Zhang et al. [30] extended this idea and proposed to use respectively a weighted arithmetic mean template or a weighted geometric mean template. The several variants proposed by Zhang et al. eliminated the noise and so improved the segmentation accuracy. However, the probability window size had to be chosen manually and was fixed during the processing.

The paper proposes an improved model, the Rayleigh Mixture Model with Neighbourhood in- 
formation (RMMN) for US image segmentation. The proposed technique incorporates neighbour information to both prior and conditional probability by means of a weighted arithmetic mean template. Moreover, the size of this probability window is adapted according to the local gradient distribution. In our opinion, RMMN will have at least the following advantages: (1) it effectively improves the classification accuracy since the Rayleigh distributions are well adapted for US images; (2) it reduces the computation time because the proposed model has fewer parameters to be estimated.

The paper is organized as follows. In Section 2, the mathematical background of RMM and EM algorithm for parameter learning is presented. In section 3, the RMMN which incorporates the mean template and adaptive window size is described in detail. Section 4 provides the experimental results and some concluding remarks are given in Section 5.

\section{Rayleigh Mixture Model for Ultrasound Image Characterizations: Mathematical Background}

\section{1. $R M M$ (Rayleigh distributions Mixture Model)}

The general FMM framework is summarized as follows. A description of the symbols used to describe the mathematical model is shown in Table 1.

\begin{tabular}{|c|l|}
\hline Symbol & description \\
\hline \hline$y_{i}$ & The $i$-th pixel of an image \\
\hline$N$ & The number of pixels in an image \\
\hline$\Omega_{j}$ & The $j$-th class in an image \\
\hline$L$ & The number of classes in an image \\
\hline$N_{i}$ & The neighbourhood pixels of the $i$-th pixel \\
\hline$p\left(y_{i} \mid \tau_{j}\right)$ & The Rayleigh distribution \\
\hline$\tau_{j}$ & The covariance of $p\left(y_{i} \mid \tau_{j}\right)$ \\
\hline$\pi_{j}$ & The prior probability of all pixel belonging to $\Omega_{j}$ \\
\hline$\gamma_{i, j}$ & The posterior probability of all the pixel $y_{i}$ belonging to $\Omega_{j}$ \\
\hline$\tau i j$ & The covariance of $p\left(y_{i} \mid \tau_{j}\right)$ by incorporating neighbour \\
\hline$\pi i j$ & $\begin{array}{l}\text { The prior probability of all pixel belonging to } \Omega_{j} \text { by incorporating neighbour } \\
\text { information }\end{array}$ \\
\hline$R_{i}$ & The normalized factor of the mean template \\
\hline
\end{tabular}

Table 1 List of the symbols used in the paper.

Let $Y=\left\{y_{1}, y_{2}, \cdots, y_{N}\right\}$ be a set of $N$ pixel intensities of a given region of interest from one image. The main objective is to cluster a dataset of $N$ pixels into $L$ labels, where the pixels from the same class will have the same label. The conditional probability distribution of the pixel $y_{i}$ corresponding to the $j$ th label is denoted by $p\left(y_{i} \mid \Omega_{j}\right)$, which is governed by the set of parameters $\Omega_{j}$. Pixel intensities are considered as a random variable, which is described by the following mixture of $L$ distributions:

$$
p\left(y_{i} \mid \Psi\right)=\sum_{j=1}^{L} \pi_{j} p\left(y_{i} \mid \Omega_{j}\right)
$$


where $y_{i}$ denotes the $i$ th pixel of the image, $\Psi=\left\{\pi_{1}, \pi_{2} \cdots \pi_{L}, \Omega_{1}, \Omega_{2} \cdots \Omega_{L}\right\}$ is the parameters set of the FMM, $\pi_{j}$ denotes the prior distributions satisfying $\sum_{j=1}^{L} \pi_{j}=1,0 \leq \pi_{j} \leq 1$. In some related works, $\pi_{j}$ is also called the coefficient of the $j$ th component in the mixture model. As shown in (2.1), the mixture model relies on $p\left(y_{i} \mid \Omega_{j}\right)$ to model the underlying distributions. Various FMM based methods differ in the used distributions to model the data. In US images application, the intensity distributions have been demonstrated to be Rayleigh distributions due to the speckle [18, 24] (Fig. 1).

The Rayleigh probability density function (PDF) $p\left(y_{i} \mid \Omega_{j}\right)$ corresponding to label $j$, is given by:

$$
p\left(y_{i} \mid \Omega_{j}\right)=p\left(y_{i} \mid \tau_{j}\right)=\frac{y_{i}}{\tau_{j}^{2}} \exp \left(-\frac{y_{i}^{2}}{2 \tau_{j}^{2}}\right)
$$

where $\tau_{j}$ denotes the covariance of the Rayleigh probability density function. Thus, $p\left(y_{i} \mid \Psi\right)$ of the RMM can be written as:

$$
p\left(y_{i} \mid \Psi\right)=\sum_{j=1}^{L} \pi_{j} p\left(y_{i} \mid \tau_{j}\right)
$$

where $\Psi=\left\{\pi_{1}, \pi_{2}, \cdots, \pi_{L}, \tau_{1}, \tau_{2}, \cdots, \tau_{L}\right\}$ denotes the set of parameters that includes the coefficients $\left\{\pi_{1}, \pi_{2}, \cdots, \pi_{L}\right\}$ and the covariances $\left\{\tau_{1}, \tau_{2}, \cdots, \tau_{L}\right\}$.

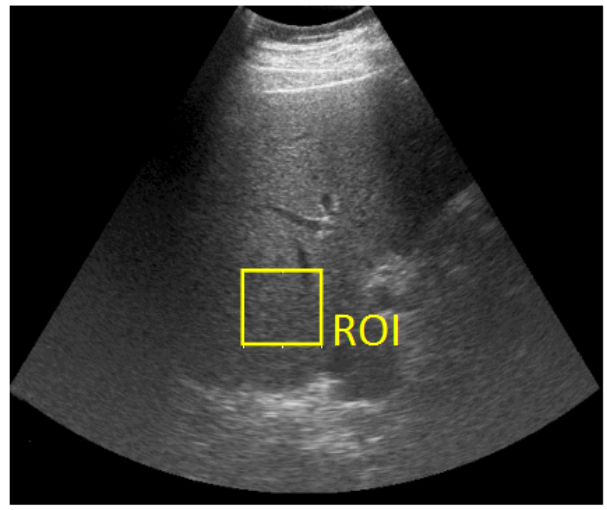

(a)

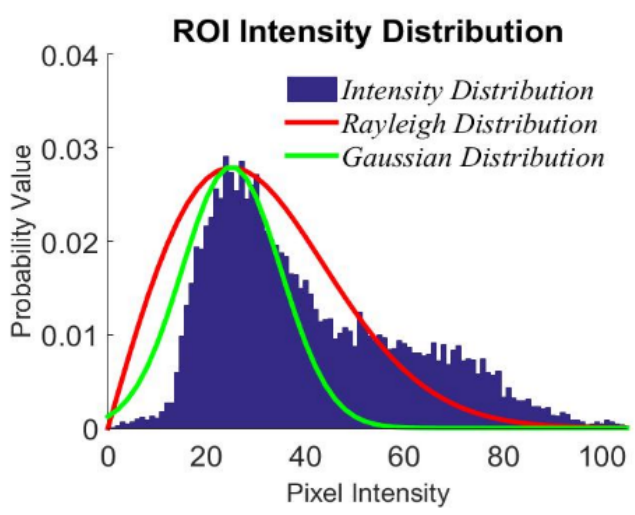

(b)

Fig. 1. The histogram of an US abdominal image, (a): original image, (b): histogram of the ROI.

\subsection{EM algorithm}

EM is most used algorithm to estimate the set of parameters. The log-likelihood function is expressed as:

$$
l(Y, \Psi)=\log p(Y, K \mid \Psi)=\sum_{i=1}^{N} \log \sum_{j=1}^{L} \pi_{j} p\left(y_{i} \mid \tau_{j}\right)
$$

The parameters set $\Psi=\left\{\pi_{1}, \pi_{2}, \cdots, \pi_{L}, \tau_{1}, \tau_{2}, \cdots, \tau_{L}\right\}$ is estimated by maximizing this likelihood function: 


$$
\Psi=\arg \max _{\Psi} l(Y, \Psi)
$$

A hidden variable $K=\left\{k_{i}\right\}$ is defined, $k_{i} \in\{1,2, \cdots, L\}$, where $k_{i}=j$ denotes that the probability $p\left(y_{i} \mid \tau_{j}\right)$ is generated by the $j$ th component. (4) becomes:

$$
l(Y, K, \Psi)=\log p(Y, K \mid \Psi)=\sum_{i=1}^{N} \log p\left(y_{i} \mid \tau_{k_{i}}\right)+\log p\left(k_{i} \mid \tau_{k_{i}}\right)
$$

However, the maximization of (6) is still difficult when the hidden parameters set $K$ is not known. EM algorithm is an iterative process which updates the parameters until convergence. It contains two steps: the Expectation step (E-step) which computes the expected value of the loglikelihood function, while the Maximization step (M-step) is to update the parameters set in order to maximize the expectation.

In the E-step, the expectation with respect to $K$ is computed as follows [12]:

$$
Q=E_{K}[l(Y, K, \Psi) \mid Y, \Psi]=\sum_{i=1}^{N} \sum_{j=1}^{L} \gamma_{i, j}\left[\log p\left(y_{i} \mid \tau_{j}\right)+\log \pi_{j}\right]
$$

where $\gamma_{i, j}$ denotes the posterior distribution of the observed variables, which is defined as:

$$
\gamma_{i, j}=\frac{\pi_{j} p\left(y_{i} \mid \tau_{j}\right)}{\sum_{k=1}^{L} \pi_{k} p\left(y_{i} \mid \tau_{k}\right)}
$$

and it satisfies the constraint $\sum_{j=1}^{L} \gamma_{i, j}=1$.

In the M-step, the parameters $\pi_{j}$ and $\tau_{j}$ can be updated by means of $Q$ :

$$
\begin{gathered}
\pi_{j}=\frac{\sum_{i=1}^{N} \gamma_{i, j}}{\sum_{i=1}^{N} \sum_{k=1}^{L} \gamma_{i, k}} \\
\tau_{j}=\frac{\sum_{i=1}^{N} \gamma_{i, j}\left(y_{i}^{2} / 2\right)}{\sum_{i=1}^{N} \gamma_{i, j}}
\end{gathered}
$$

where $\tau_{j}$ is achieved using the posterior probability $\gamma_{i, j}$ and also the pixels information $y_{i}$.

The final parameters set is obtained after convergence of the EM algorithm. The condition of convergence is generally considered as $\left|\Psi^{n+1}-\Psi^{n}\right|<\epsilon$. The stopping threshold $\epsilon$ must be small, i.e. in the proposed model it was set to be $10^{-8}$.

\section{Rayleigh Mixture Model with Spatial Information}

\subsection{Incorporating Neighbor Spatial Information}

As aforementioned in previously, Rayleigh distribution is well suited to describe the intensity distributions of the speckle in US images since it is directly related to the physics of the image formation. However, as any Finite Mixture Model, RMM is only based on the pixel intensities without any spatial information but this information is very important in image classification. Neighbourhood information is one of the most important spatial information. In fact, the classification probabilities assigned to the neighbourhoods pixels should affect the probabilities of the current pixel. In 
order to incorporate neighbourhood information into the conventional RMM, we adopted a neighbourhood weight strategy by an adaptive weighted template to ensure the conditional probability of each pixel belonging to $\Omega_{j}$ class to be affected by the neighbours. Inspired by the method introduced in [30], the neighbourhood class distribution information is defined as a weighted template that impacts the current class distribution. We defined the neighbourhood weighted probability as:

$$
p\left(y_{i} \mid \Psi\right)=\sum_{j=1}^{L} \pi_{i j} p\left(y_{i} \mid \tau_{i j}\right)
$$

The main difference to (3), is that, $\pi_{j}$ is changed to $\pi_{i j}$ that denotes that the prior distribution of pixel $y_{i}$ belongs to class $j$. We also adopted a weight window for the conditional distribution probabilities:

$$
p\left(y_{i} \mid \Psi\right)=\sum_{j=1}^{L} \pi_{i j}\left(\sum_{m \in N_{i}} \frac{\omega_{m}}{R_{i}} p\left(y_{m} \mid \tau_{i j}\right)\right)
$$

Where $N_{i}$ denotes the neighbourhood window of the current pixel, $\omega_{m}$ is the weight associate to pixel $y_{m}, m \in i$, and $R_{i}$ is a normalizing factor which is the sum of the pixels in $N_{i}$ including the current pixel. In our model, the EM algorithm was also used for the parameters estimation.

In the E-step, the expectation $Q$ was calculated as:

$$
Q=\sum_{i=1}^{N} \sum_{j=1}^{L} \gamma_{i, j}\left[\sum_{m \in N_{i}} \frac{\omega_{m}}{R_{i}} \log p\left(y_{m} \mid \tau_{j}\right)+\log \pi_{i j}\right]
$$

where the posterior distribution $\gamma_{i, j}$ was modified as:

$$
\gamma_{i, j}=\frac{\pi_{i j} \sum_{m \in N_{i}} \frac{\omega_{m}}{R_{i}} p\left(y_{m} \mid \tau_{i j}\right)}{\sum_{h=1}^{L} \pi_{i h} \sum_{m \in N_{i}} \frac{\omega_{m}}{R_{i}} p\left(y_{m} \mid \tau_{i h}\right)}
$$
lows:

In the M-step, the parameter $\tau_{i j}$ that incorporates neighbour information was estimated as fol-

$$
\tau_{i j}=\frac{\sum_{i=1}^{N} \gamma_{i, j} \frac{\omega_{m}}{R_{i}}\left(y_{i}^{2} / 2\right)}{\sum_{i=1}^{N} \gamma_{i, j}}
$$

For a specific weight window, the prior probability $\pi_{i j}$ was written as follows:

$$
\pi_{i j}=\frac{\sum_{m \in N_{i}} \omega_{m} \gamma_{m, j}}{\sum_{h=1}^{L} \sum_{m \in N_{i}} \omega_{m} \gamma_{m, h}}
$$

From (13) to (16), $\omega_{m}, N_{i}$, and $R_{i}$ are the same denotations as defined in (12), $\gamma_{i, j}$ denotes the posterior probability as defined in (8).

The EM algorithm was processed iteratively until convergence. Notice that there are now two parameters $\pi_{i j}$ and $\tau_{i j}$ to be estimate in the M-step.

\subsection{Adaptive window size}

As seen in (12), the incorporation of neighbour spatial information is directly related on the neighbourhood window $N_{i}$ around the current pixel with $\omega_{m}$ the probability weights. In this subsection, 
we will discuss about the properties and definition of this probability window. The probability window manages the neighbour information. The simplest probability window choice is the mean template: the probability window size is fixed and chosen as $3 \times 3$ and all elements in $N_{i}$ are equal to $1, \omega_{m}=, m \in N_{i}$. However, such a fixed window size is not satisfactory in the sense that we expect to integrate more information in homogeneous regions and, in counterpart, to decrease the window size on the region boundaries in order to gain accuracy on the region border estimation.

Locally, the distribution of the pixels' gradient magnitude distribution reflects the image homogeneity: gradient magnitude values are small or basically equals in homogeneous region whereas are high in inhomogeneous regions. We propose to adapt the window size according on the local gradient magnitude distribution. For a specific pixel, the windows size will be estimated as follows. Let us begin with a small $3 \times 3$ window size. The mean and the variance of the gradient magnitude of the pixels inside the window reflect the local homogeneity or in-homogeneity. The window size is then expanded while the mean and the variance of all elements inside the window are less than a given threshold.

In more details, the procedure of the adaptive window size estimation is as follows:

Step 1: Initialize the window size to $3 \times 3$, initialize the iterative step to $d=0$.

Step 2: Update $d=d+1$.

Expand the probability window size to $(3+d \cdot 2) \times(3+d \cdot 2)$.

(a) Compute the mean value $G_{l o c-m e a n}$ of the gradients magnitude of the pixels inside the window. If $G_{l o c-m e a n}<T_{\text {mean }}\left(T_{\text {mean }}\right.$ is a manual defined fixed threshold), go to step 2; else, go to (b).

(b) Compute the variance $G_{l o c-v a r}$ of the gradients magnitude of the pixels inside the window.: if $G_{l o c-v a r}<T_{v a r}$ ( $T_{v a r}$ is a manual defined fixed threshold), go to step 2; else, go to step 3.

Step 3: Shrink the probability window to $(n-d \cdot 2) \times(n-d \cdot 2)$. In this window set $\omega_{m}=1$, $m \in N_{i}$.

This process can be performed once before the EM algorithm. First a global gradient magnitude map $G_{\text {global }}$ is computed for the entire image. Then the windows size is estimated from $G_{\text {global }}$ for each pixel and stored in memory before to be used in the EM algorithm.

\subsection{Complete algorithm}

In summary the main procedure of the proposed mixture model is as follows:

Step 1: Initialization

Initialize the parameters set $\Psi$, the covariance values $\tau_{i j}$, and the prior distributions $\pi_{i j}$.

Step 2: Window size estimation.

(a) Compute the global gradient magnitude map $G_{\text {global }}$.

(b) Estimate and store the window size for each pixel using the information of $G_{\text {global }}$.

Step 3: E-step.

Evaluate the posterior distribution $\gamma_{i j}$ in (14) using the current parameters set.

Step 4: M-step.

(a) Update the covariance values $\tau_{i j}$ by using (15).

(b) Update the prior distributions $\pi_{i j}$ by using (16).

Step 5: Evaluate the expectation value $Q$ in (13) and check the convergence of either the expectation value or the parameter values. If the convergence is reached, finish; else go to step 3. 


\section{Experiments and discussions}

In this section, several experiments have been conducted on synthetic and real ultrasound data. The experiments were performed on an Intel i5 Core $2.8 \mathrm{GHz}$ CPU with 12.0 GB RAM, with MATLAB R2013a.

\subsection{Experiments on Synthetic Data}

Two kinds of synthetic US images with progressive anatomical realism were used. The first image was composed of an elliptically-shaped region within a background region (Fig. 2 (a)), called Elliptical Image in the rest of the paper. The second was simulated from a segmented CT image of a human abdomen including the liver and a kidney (Fig. 2 (b)), called Abdomen Image. Each tissue (region) of these images was characterized by a specific acoustic impedance and a spatial distribution of scatterers. The simulated method predicted the appearance and properties of a BScan US image from a probe model and these region parameters [31]. The parameters dened in [31] were used for the simulated Abdomen Image. For the Elliptical Image, we assigned to the elliptic and the background region the parameters dened in [31] for liver and fat, respectively. For both images, we simulated an US image acquired with a C2-5 circular US scanning 3.5 MHz probe. In these images, the greyscale depended only on the spatial distribution of the scatterers. The image size was $1352 \times 1149$ for both images. On all the methods, the processing was performed on the available information inside the US beam only. The ground truth of these two images is shown in Fig. 2 (c) and (d).

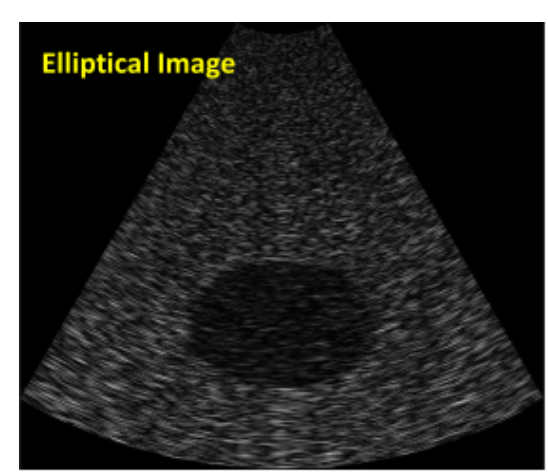

(a)

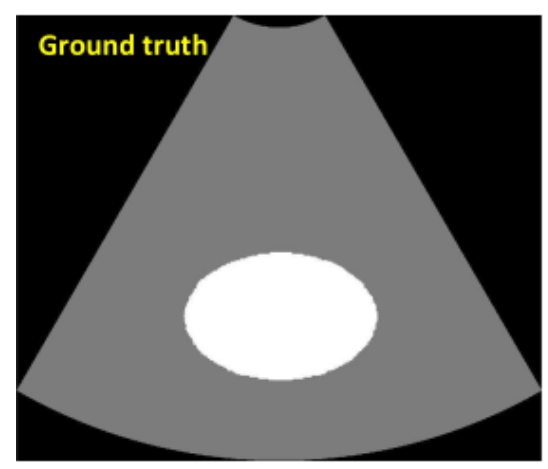

(c)

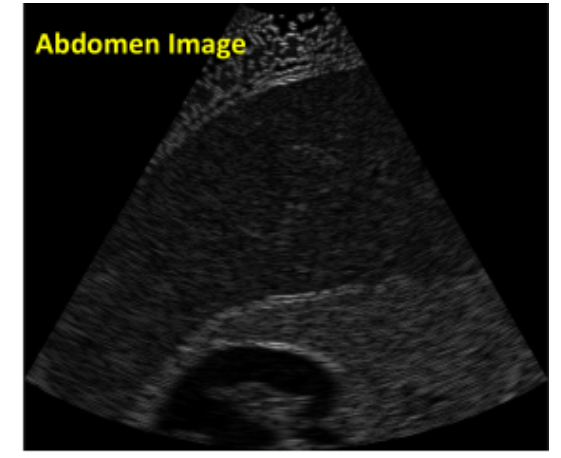

(b)

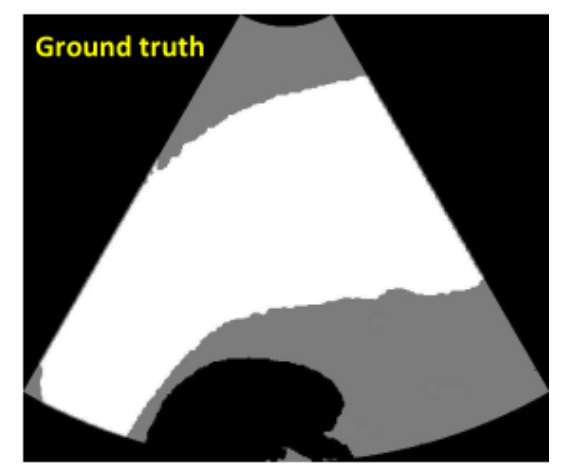

(d)

Fig. 2. (a): Elliptical Image, $(b)$ : Abdomen Image, $(c)$ : Ground truth of Elliptical Image, $(d)$ : Ground truth of Abdomen Image. 
We have compared our proposed RMMN model to two conventional FMMs without neighbourhood information: Gaussian Mixture Model (GMM) and Rayleigh Mixture Model (RMM), and to the two methods which are considered as references when incorporating spatial information: Hidden Markov Random Field model with Fuzzy C-Means clustering (HMRF-FCM) [28], and the incorporation of an arithmetic mean template to both the conditional and prior probabilities to a Gaussian Mixture Model (ACAP) [30].

The presentation of the model mixture on $2 \mathrm{D}$ data is difficult. We proposed to present the distribution as a colour image. First we assigned a specific colour $C_{k}$ to each class $k$ of our model. For each pixel, the FMM was then encoded by the following colour composition formula:

$$
C\left(y_{i}\right)=\sum_{k=1}^{L} C_{k} p\left(k \mid y_{i}, \Omega_{k}\right)
$$

where $C\left(y_{i}\right)$ is the specified colour assigned to the $i$ th pixel and $C_{k}$ is the colour assigned to the $k$ th class.

For Elliptic Image, we set the number of classes to be $L=2$. The performances of the different methods including HMRF-FCM, GMM, ACAP, RMM, and RMMN are illustrated in Fig. 3 (a)(e). On these images the pixel colours are given using (17) with green for 'background' and red for 'elliptic region'. Fig. 3 (a) presents the segmentation by HMRF-FCM which is blurry since oversmooth. Fig. 3 (b) and (c) present respectively the classification by GMM and ACAP. It can be noticed that the contour of the ellipse is not smooth. Some pixels on the border are misclassified. The incorporated neighbourhood information by ACAP seems not improving GMM in the US image context. Fig. 3 (d) and (e) present the results by RMM and RMMN, respectively. The boundary is much better defined using RMM than GMM as shown in Fig. 3 (b) and (c). The result on RMMN (Fig. 3 (e)) proves that the incorporation of neighbourhood information in the RMM scheme improves significantly the classification. It is shown that Rayleigh Mixture Model with spatial information is superior to the other methods.

Fig. 4 shows the results of the different methods applied on Abdomen image. Two classes $(L=2)$ are considered in this experiment, one class indicated the liver using red while the other class indicated the non-liver (mainly fat and a muscle) using green instead. Fig. 4 (b) and (d) present the classification results of the two models without spatial information, GMM and RMM, respectively. We can see that there are some misclassified pixels either inside the tissue or on the border. The accuracy of the classification results achieved by the models with spatial information (HMRF-FCM, ACAP and RMMN, respectively) are better than those without spatial information. The segmentation by HMRF-FCM, as shown in Fig. 4 (a), is blurry and over-smooth. ACAP, as shown in Fig. 4 (c), is better than HMRF-FCM whereas there are still some misclassifications in the liver region. It can be noticed that the segmentation result achieved by RMMN, as shown in Fig. 4 (e), shows a more homogeneous liver region than HMRF-FCM and ACAP.

We employed the Dice Similarity Coefficient (DSC) [32] between the ground truth and the classified images in order to evaluate the accuracy of the proposed methods.

$$
D S C=\frac{2\left|R_{s} \cap R_{g}\right|}{\left|R_{s}\right|+\left|R_{g}\right|}
$$

where $R_{s}$ denotes the target region of the classified images, $R_{g}$ denotes the target region of the ground truth and $|\bullet|$ the number of pixels of the regions. The ground truths are shown in Fig. 2 (c, d). The DSC returns a value between 0 and 1 , with 0 indicating a total miss-classification and with 1 indicating a perfect pixel classification. 


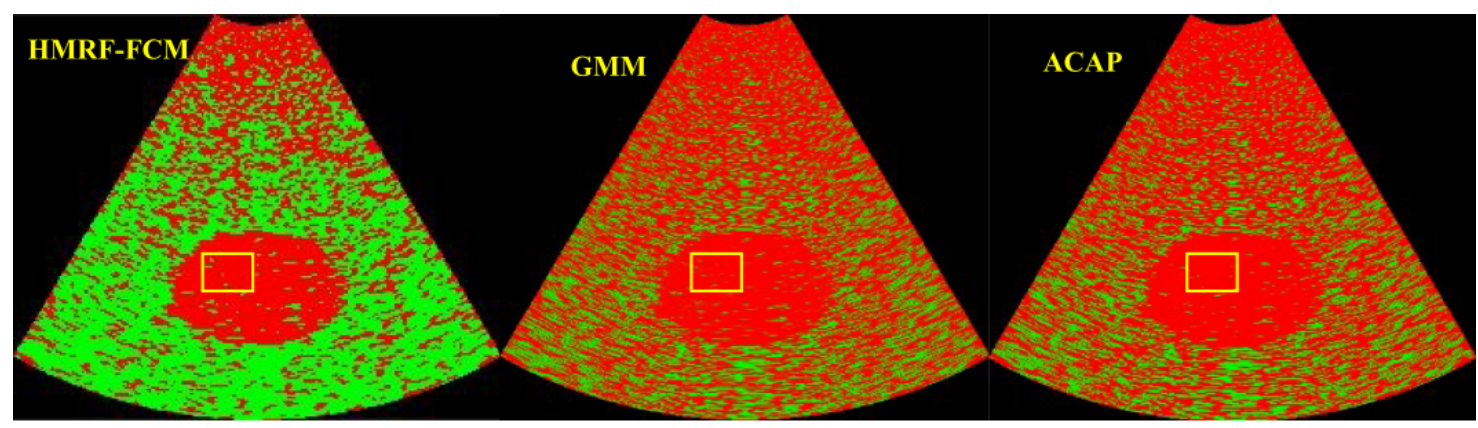

(a)

(b)

(c)

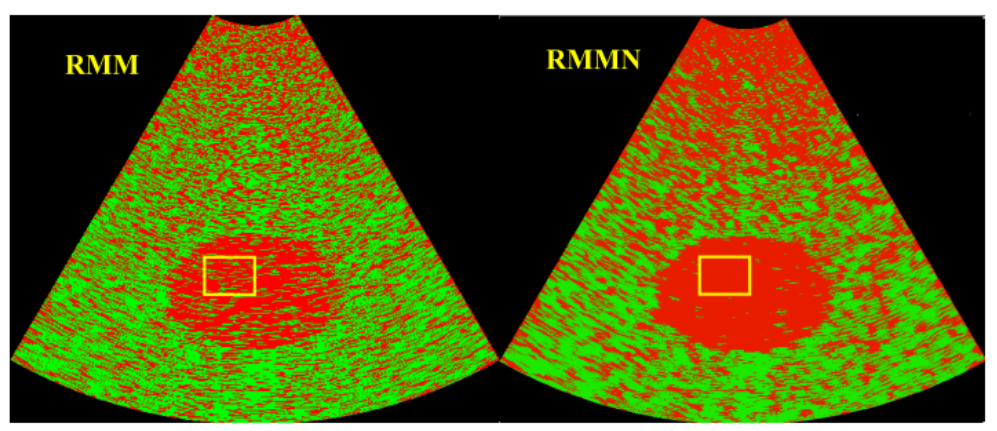

(d)

(e)

Fig. 3. Classification results on Elliptic Image, (a): by HMCR-FCM, (b): by GMM, (c): by ACAP, (d): by RMM, (e): by RMMN.

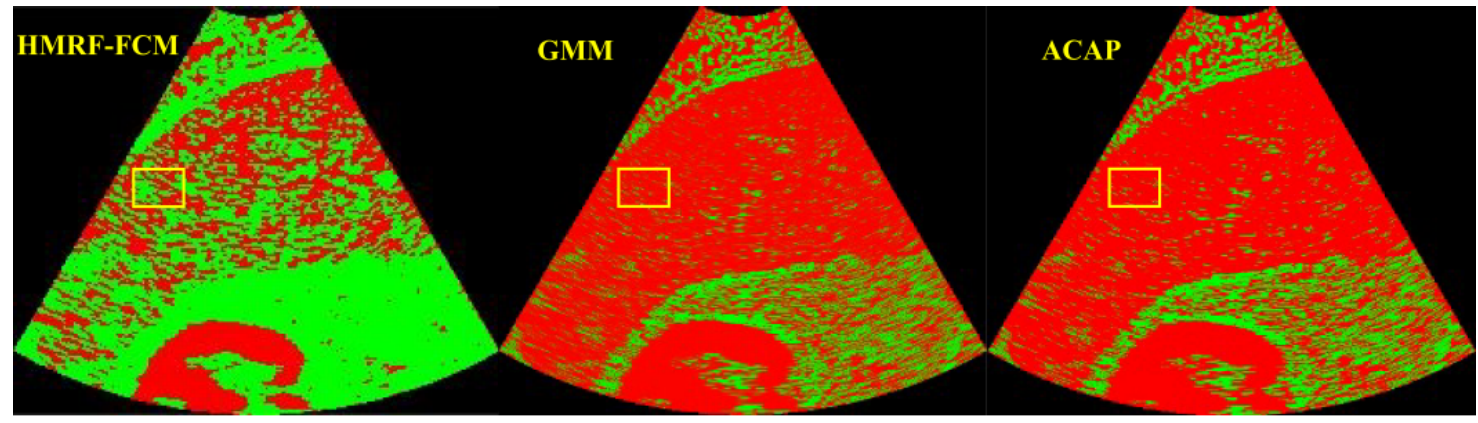

(a)

(b)

(c)

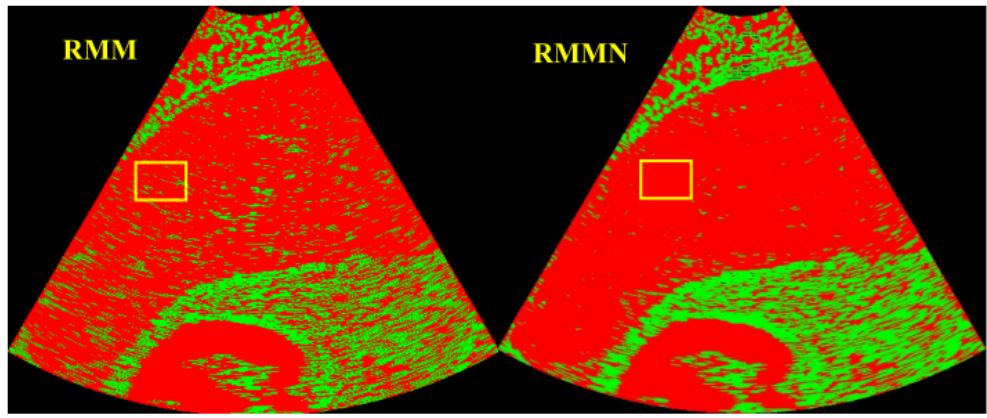

(d)

(e)

Fig. 4. Classification results on Abdomen Image, (a): by HMRF-FCM, (b): by GMM, (c): by ACAP, $($ d): by RMM, (e): by RMMN. 
The DSCs shown in Table 2 confirm the previous qualitative evaluation. Compared to other methods, the proposed method yields the best segmentation results with the highest DSC values. On the Elliptical Image, there is $10.5 \%$ difference on DSC between ACAP and RMMN, this indicates that Rayleigh mixture models are much suitable for characterizing US image than Gaussian mixture models. The $2.8 \%$ difference between RMM and RMMN indicates that incorporating spatial information improves the segmentation accuracy. However, the DSCs are relatively low, mainly because the speckle size changes from the top to the bottom of the image, the intensity distributions in the same region is not the same from the top to the bottom of the image. This distribution shift is difficult to handle by a global mixture model so lots of pixels are misclassified on the top of the image.

\begin{tabular}{|c|c|c|c|}
\hline & Elliptical Image & Abdomen Image & Mean of Total Images \\
\hline \hline HMRF-FCM & 0.275 & 0.544 & 0.410 \\
\hline GMM & 0.355 & 0.741 & 0.548 \\
\hline ACAP & 0.355 & 0.746 & 0.551 \\
\hline RMM & 0.425 & 0.758 & 0.592 \\
\hline RMMN & 0.454 & 0.764 & 0.609 \\
\hline
\end{tabular}

Table 2 Dice Similarity Coefficient (DSCs) indicating the segmentation accuracy using the several models.

We also evaluate the computation time cost of these methods for comparison (Table 3). For Elliptical Image, HMRF-FCM has the highest time cost and takes $1624.1 \mathrm{~s}$ to segment the image. GMM is the sub-slowest method which takes $301.6 \mathrm{~s}$. ACAP, RMM and RMMN take $55.5 \mathrm{~s}, 14.2$ $\mathrm{s}$ and $13.6 \mathrm{~s}$, respectively. The computation time of RMMN is sharply reduced and is nearly $1 \%$ of HMRF-FCM, 5\% of GMM and 24\% of ACAP. For Abdomen Image, HMRF-FCM takes 1584.5 $\mathrm{s}$ to segment the image. GMM still is the sub-slowest method which takes $267.6 \mathrm{~s}$. ACAP, RMM and RMMN take $51.0 \mathrm{~s}, 14.1 \mathrm{~s}$ and $13.7 \mathrm{~s}$, respectively. The difference between GMM and RMM can be explained by the fact that only two parameters $\left\{\pi_{i j}, \tau_{i j}\right\}$ have to be estimated in a Rayleigh distribution while there are three parameters $\left\{\pi_{i j}, \mu_{i j}, \Sigma_{i j}\right\}$ in a Gaussian distribution as shown in (13)-(16). Compared to GMM, RMM avoids computing the time consuming covariance matrix $\Sigma_{i j}$. More surprising, RMMN takes less computation time than RMM. The computation time loss used to incorporate the neighbour information seems to be compensated by a faster convergence. In conclusion the proposed model is the most efficient since it has fewer parameters and an adaptive window size.

\begin{tabular}{|c|c|c|c|c|}
\hline & Elliptical Image (s) & $\begin{array}{c}\text { Percent based } \\
\text { on HMRF-FCM }\end{array}$ & Abdomen Image (s) & $\begin{array}{c}\text { Percent based } \\
\text { on HMRF-FCM }\end{array}$ \\
\hline \hline HMRF-FCM & 1624.1 & - & 1584.5 & - \\
\hline GMM & 301.6 & $18.57 \%$ & 267.6 & $16.89 \%$ \\
\hline ACAP & 55.5 & $3.41 \%$ & 51.0 & $3.22 \%$ \\
\hline RMM & 14.2 & $0.87 \%$ & 14.1 & $0.89 \%$ \\
\hline RMMN & 13.6 & $0.84 \%$ & 13.7 & $0.87 \%$ \\
\hline
\end{tabular}

Table 3 Computation time cost of synthetic images using several models. 


\subsection{Experiments on real US data}

We evaluated also our method on real US images used to guide High-Intensity Focused Ultrasound (HIFU) therapies. HIFU ablation is considered as a safe, feasible and cost-effective alternative for the treatment of many diseases. Since HIFU is a non-invasive therapeutic procedure, it is applied for women patients with symptomatic uterine fibroids. In the middle of the HIFU probe, there is an US imaging device which is used to guide the therapy. These US images are used in a preoperative stage to estimate the fibroid location and in an intra-operative stage to estimate the limits of the fibroid in order to position the HIFU focal ablation spots over the lesion. During HIFU operation, the surgeon has to delineate manually the fibroid leading to a long processing time during which the patients has to wait in the treatment apparatus. An effective and efficient fibroid characterization method will help to reduce the doctors burden and relieve patients pain.

We worked on 55 women patients to evaluate the proposed method. We also make comparison for HMCR-FCM, GMM, ACAP, RMM, and RMMN. For each method, we initialized the number of classes to $3(L=3)$. We examined the results qualitatively. Fig. 5 presents the segmentation results on 3 representative patients among the 55 women patients. One line represents a patient with (from left to right) the original uterine US images and segmentation results of HMRF-FCM, GMM, ACAP, RMM and RMMN, respectively. The colours of the segmentation images are set according to (17) with the $C_{k}$ s set to blue, green and red.

The upper line of Fig. 5 shows Patient 1 with a small uterine fibroid beside the bladder. Uterine fibroid is the target in HIFU therapy. The uterine fibroid is easy to locate since the bladder presents a hypoechogeneic (shadow) region. However, the blurred boundary of the uterine fibroid increases the localisation complexity. The segmentation result obtained using HMRF-FCM shows a homogeneous region for the bladder and the uterine fibroid. The boundary of the uterine fibroid is relatively well defined. GMM cannot distinguish the fibroid. ACAP can distinguish the fibroid but still blurs the bladder boundary. RMM has a better estimation of the fibroid boundary than ACAP but there are a number of fragments inside the uterine. RMMN shows a more homogeneous region and a clear boundary of the uterine fibroid.

The middle line of Fig. 5 shows Patient 2 with a big uterine fibroid. Compared to Patient 1, the uterine fibroid is more complex to locate since there is no external reference. The segmentation result using HMRF-FCM shows a homogeneous region and a clear boundary of the uterine fibroid. There are many fragments inside uterine and blurred boundaries in the segmentation results obtained using GMM, ACAP, and RMM. RMMN presents a homogeneous region of the uterine fibroid and a clear boundary.

The lower line of Fig. 5 shows Patient 3 with an irregular uterine fibroid. There is an artefact beside the uterine fibroid which makes it difficult to achieve its location. The segmentation result using HMRF-FCM shows a homogeneous uterine fibroid region and the artefact can be distinguished. The results of GMM, ACAP, and RMM present a number of fragments in the artefact region and a bad definition of the uterine fibroid boundary. The segmentation result of RMMN is in the same level of accuracy as HMRF-FCM.

This visual inspection shows that the results obtained by GMM, ACAP and RMM are really inhomogeneous. These results are highly fragmented and present also blurry boundaries. The presence of tinny fragments near the boundaries can disturb the estimation of the fibroids area needed by the HIFU therapy.

Some texture analysis features as the Homogeneity (also called the'Inverse Difference Moment") of grey-level co-occurrence matrix analysis [33] are directly related to the level of homogeneity. In order to quantitatively check our homogeneity ascertainment, we tried to compare the 


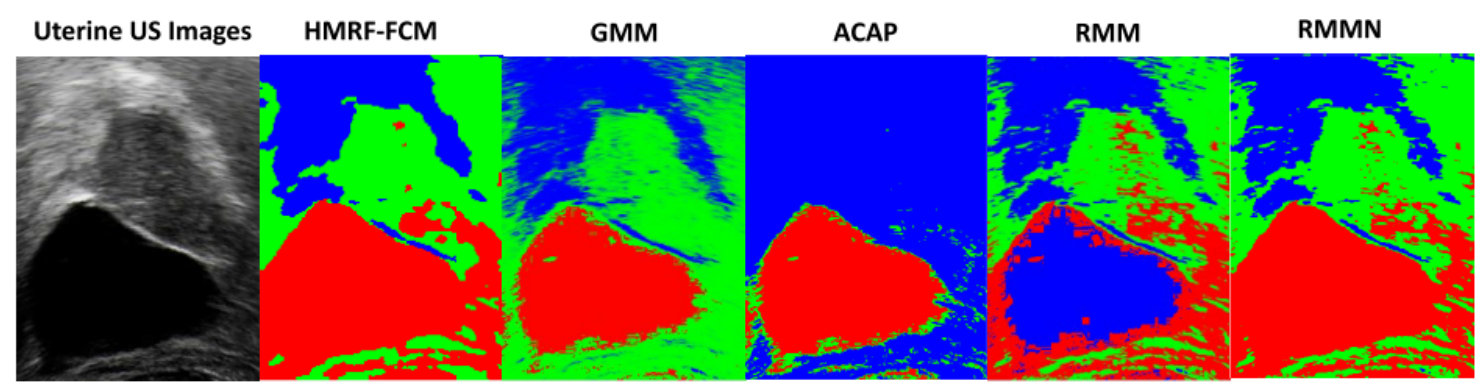

(a) Patient 1

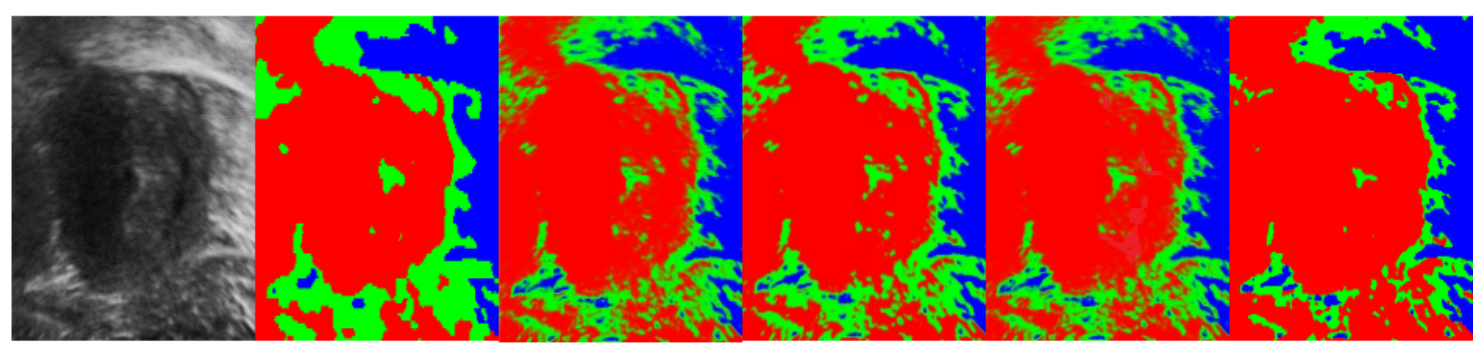

(b) Patient 2

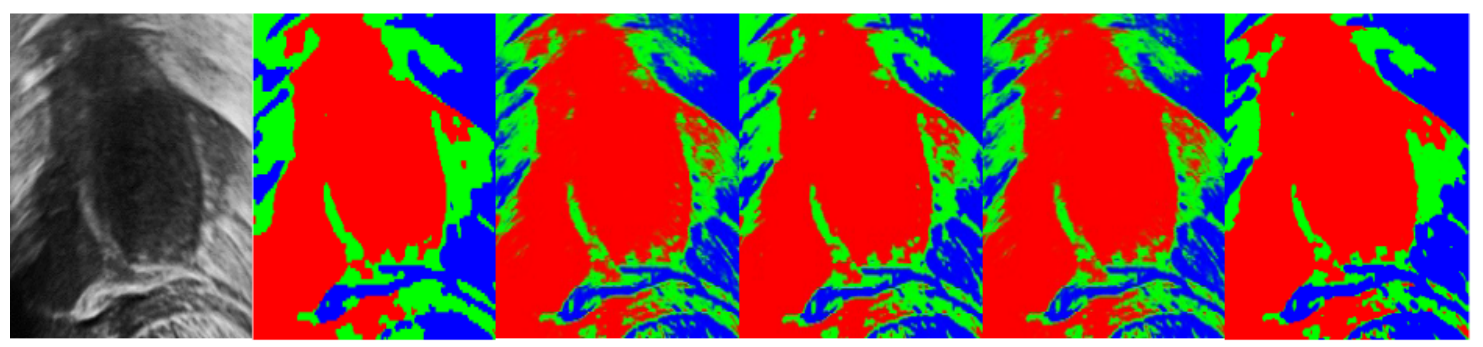

(c) Patient 3

Fig. 5. Results on real uterine US images. The first column: uterus US images from 3 patients. The segmentation results of HMRF-HCM, GMM, ACAP, RMM, and RMMN are shown from the second column to the sixth column. 
mean Homogeneity feature measured inside the fibroid between the several methods (Table 4). As shown in Table 4, HMRF-FCM has the highest average Homogeneity (0.968) inside the fibroid. GMM and RMM which does not take spatial information take the lowest one $(\approx 0.93)$. ACAP achieves 0.94. RMMN takes almost the same value as HMRF-FCM.

\begin{tabular}{|c|c|c|c|c|c|}
\hline \multirow{2}{*}{ Models } & \multicolumn{4}{|c|}{ HIFU images } & Mean value on \\
& Patient 1 & Patient 2 & Patient 3 & Patient 4 & all the images \\
\hline \hline HMRF-FCM & 0.97 & 0.97 & 0.96 & 0.97 & 0.968 \\
\hline GMM & 0.93 & 0.94 & 0.93 & 0.92 & 0.93 \\
\hline ACAP & 0.94 & 0.95 & 0.94 & 0.93 & 0.94 \\
\hline RMM & 0.94 & 0.94 & 0.93 & 0.92 & 0.932 \\
\hline RMMN & 0.96 & 0.97 & 0.96 & 0.98 & 0.967 \\
\hline
\end{tabular}

Table 4 Homogeneiy features measured on grey-level co-occurrence matrices using several models on real US data

From the above-mentioned results, it is shown that incorporating spatial information into the Rayleigh distributions mixture model provides more homogeneous regions. The segmentation results using HMRF-FCM and our proposed method give more homogeneous regions on the uterine. Such homogeneous regions are needed to improve the therapy accuracy of the HIFU treatment.

However, it can be clearly seen that none of these methods can be self-sufficient to perform alone the segmentation on US image. Even if in the best case, the results are too inhomogeneous to provide a direct segmentation of the data. Also, even if theoretically the speckle should follow a Rayleigh distribution, and so be better characterized by RMM and RMMN, it should be noticed that the real US images given by the ultrasound equipment are the results of some hidden postfiltering (attenuation compensation, "noise cancelling" and so on). Usually, the speckle on the end-user image no more follows a Rayleigh distribution. Nevertheless, we think that the ultrasound manufacturers could apply our method in the US treatment pipeline before these post-processing filters in order to be adjusted adequately to the characteristics of the data.

We also evaluated the computation time cost of the several methods applied on the real US data (Table 5). As on the simulated data, HMRF-FCM has the highest computation time cost and takes a mean time of $113.9 \mathrm{~s}$ to segment an image. GMM is the sub-slowest method which takes $2.6 \mathrm{~s}$. ACAP takes $2.7 \mathrm{~s}$ that is much faster than GMM. RMM and RMMN take both almost $0.8 \mathrm{~s}$. The computation time cost of RMMN is sharply reduced compared to the other methods and is nearly $0.7 \%$ of HMRF-FCM and $30 \%$ of GMM and ACAP.

\begin{tabular}{|c|c|c|c|c|c|c|}
\hline \multirow{2}{*}{ Models } & \multicolumn{4}{|c|}{ US images } & Mean time on & $\begin{array}{c}\text { Percent based } \\
\text { all the images }\end{array}$ \\
\cline { 2 - 5 } & Patient 1 & Patient 2 & Patient 3 & Patient 4 & 113.9 & - \\
\hline \hline HMRF-FCM & 130.7 & 91.3 & 115.4 & 118.2 & 2.6 & $2.2 \%$ \\
\hline GMM & 2.9 & 2.1 & 2.6 & 2.7 & 2.7 & $2.3 \%$ \\
\hline ACAP & 3.0 & 2.2 & 2.6 & 2.9 & 0.8 & $0.7 \%$ \\
\hline RMM & 10.9 & 0.6 & 0.8 & 0.9 & 0.8 & $0.7 \%$ \\
\hline RMMN & 1.0 & 0.7 & 0.8 & 0.8 & 0.8 \\
\hline
\end{tabular}

Table 5 Computation time cost (in s) on real data using several models.

In conclusion, on the real images, our proposed model has almost the same segmentation results as HMRF-FCM but with a much faster computation time. This is especially interesting because 
real time is an important issue in US guided therapies. We hope that our method will be able to speed up the time used by therapists to locate uterine fibroids for HIFU therapy

\subsection{Potential usage of RMMN}

As mentioned in the literature and as we have noticed previously, FMM methods cannot be used self-sufficiently to perform the segmentation on US images. Classically, such methods are used as a pre-processing step to further treatment as region estimation using texture analysis or boundary estimation using active contours or level sets.

As an example of the potential use of our method, we incorporated the RMMN in a level set framework. Level set methods have been widely used in image processing and computer vision owing to its efficiency [34]. In our case, we tried to estimate the performance of the level set when it is applied directly to the original grey level Elliptical Image.

We applied level set method on two kinds of images: original US image and RMMN clustering image. The initializations of these two images are the same, as shown in Fig. 6 (a) and (c). When we applied on the original grey level US image directly, it can be observed on Fig. 6 (b), that the outline of the elliptic region is not reached even after 300 iterations. Comparatively when we applied on the RMMN clustering image, the level set method was able to delineate the elliptical region only after 60 iterations, as shown in Fig. 6 (d). The processing time reduced sharply by means of the initialization on the classification achieved by RMMN.

The objective of this section was not to show an accurate segmentation scheme but only to illustrate the ability of FMM methods to be integrated in more complex US image segmentation frameworks [35]. From the most used methods in US segmentation, we can for example mention texture analysis tools. These methods needs as homogeneous features as possible in order to perform the classification. We suppose that our method can provide homogeneous and significant information for the final analysis. Iterative methods as active contours or level sets are also popular in US image segmentation. Our model is homogeneous and moreover fast enough to be included in such an iterative segmentation scheme.

\section{Conclusion}

In this paper, we have proposed the Rayleigh mixture model with neighbourhood information $(\mathrm{RMMN})$ in order to characterize ultrasound (US) images. Such a model seems to be well adapted to describe US data because the speckle is known to follow a Rayleigh distribution. We proposed a method to make the Rayleigh mixture model more robust to noise or to outliers by integrating spatial information. We also improved the computation speed by integrating an adaptive window in the model. Experiments on synthetic and real US images demonstrated that our model has higher segmentation accuracy and needs lower computation time than the classical methods.

Applied to US images, the RMMN has two primary advantages: (1) the classification accuracy is promoted because of two reasons: first of all, Rayleigh distribution is more suitable to describe the physics properties of the US image; second, the incorporation of neighbourhood information induces more homogeneous regions; (2) the computation time is reduced sharply because RMMN no longer needs to calculate some time consuming covariance matrices as in Gaussian Mixture Models. The proposed model not only is attractive in clinical applications, but also can be useful as a pre-processing step in more complex US segmentation methods (texture analysis, level sets, active contours and so on). 


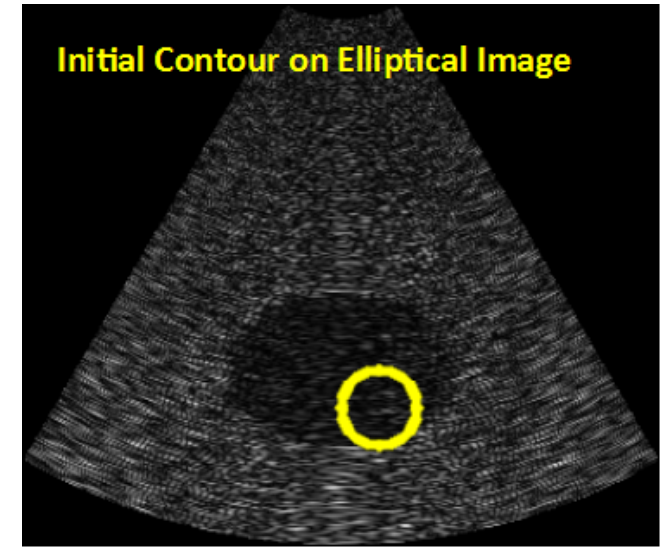

(a)

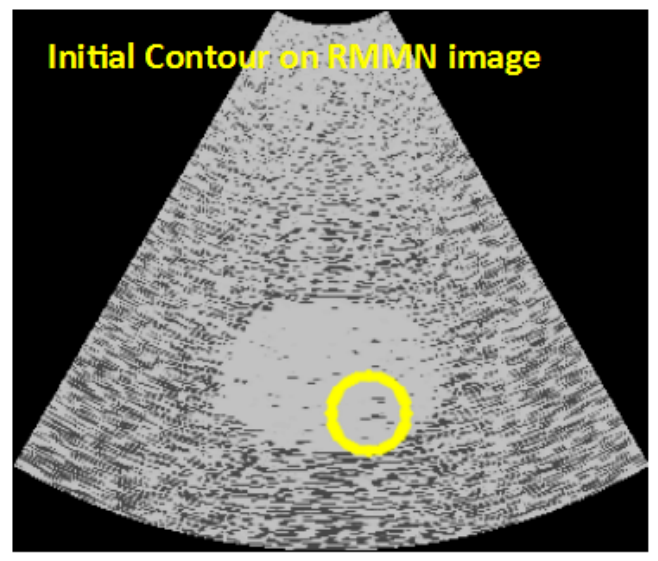

(c)

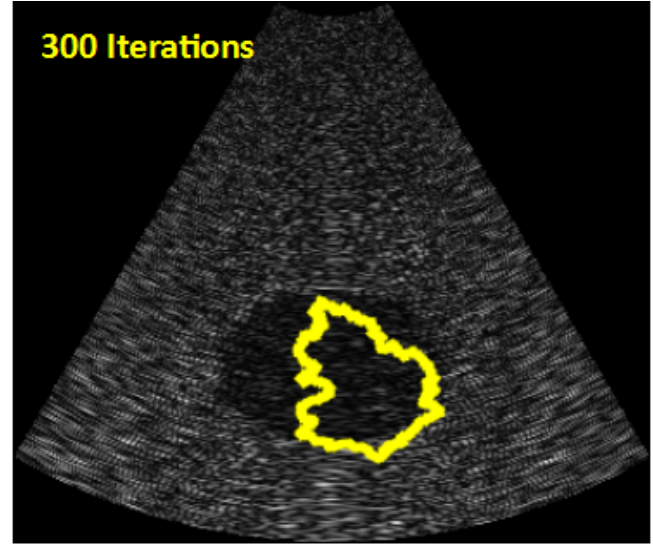

(b)

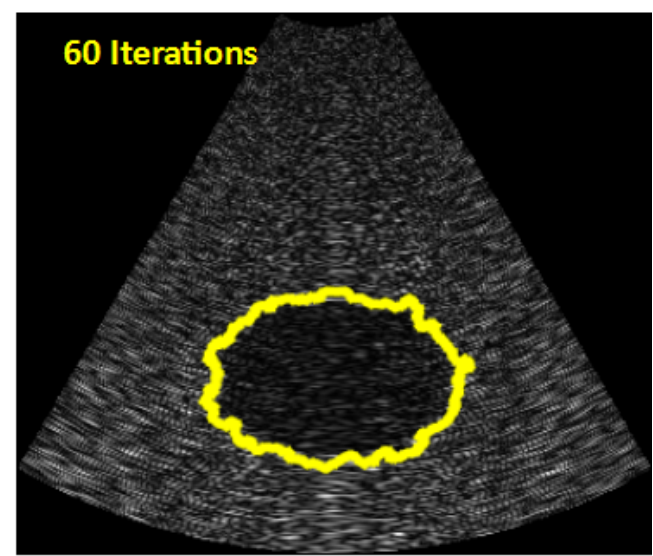

(d)

Fig. 6. Level set segmentation performed on Elliptical Image (upper row) and the classified image using RMMN (lower row), (a): level set initialization on original image, $(b)$ : results after 300 iterations, (c): level set initialization by RMMN classification, $(d)$ : results after 60 iterations. 
In the future work, the proposed method can be extended to help surgeons to locate uterus during the HIFU clinical surgery so that the operation cost time can be reduced.

\section{Acknowledgements}

This work was supported by the by the National Natural Science Foundation of China under Grants 61271312, 61201344, 61401085, 31571001, and 81530060, by the Natural Science Foundation of Jiangsu Province under Grant BK2012329, BK2012743, BK20150647, DZXX-031, BY201412711, by the 333 project under Grant BRA2015288 and by the Qing Lan Project.

\section{References}

[1] M. Sonka, V. Hlavac, and R. Boyle, Image processing, analysis, and machine vision. Cengage Learning, 2014.

[2] A. Bali and S. N. Singh, "A review on the strategies and techniques of image segmentation," in Advanced Computing \& Communication Technologies (ACCT), 2015 Fifth International Conference on. IEEE, 2015, pp. 113-120.

[3] G. McLachlan and D. Peel, Finite mixture models. John Wiley \& Sons, 2004.

[4] C. M. Bishop, Pattern recognition and machine Learning. Springer, 2006, vol. 128.

[5] N. Bouguila, "Count data modeling and classification using finite mixtures of distributions," IEEE Transactions on Neural Networks, vol. 22, no. 2, pp. 186-198, 2011.

[6] W. Fan, N. Bouguila, and D. Ziou, "Variational learning for finite Dirichlet mixture models and applications," IEEE Transactions on Neural Networks and Learning Systems, vol. 23, no. 5, pp. 762-774, 2012.

[7] H. Wei, X.-M. Wang, and L. L. Lai, "Compact image representation model based on both nCRF and reverse control mechanisms," IEEE Transactions on Neural Networks and Learning Systems, vol. 23, no. 1, pp. 150-162, 2012.

[8] S. E. Yuksel, J. N. Wilson, and P. D. Gader, "Twenty years of mixture of experts," IEEE Transactions on Neural Networks and Learning Systems, vol. 23, no. 8, pp. 1177-1193, 2012.

[9] R. Guillemaud and M. Brady, "Estimating the bias field of MR images," IEEE Transactions on Medical Imaging, vol. 16, no. 3, pp. 238-251, 1997.

[10] I. Wells, W.M., W. Grimson, R. Kikinis, and F. Jolesz, "Adaptive segmentation of MRI data," IEEE Transactions on Medical Imaging, vol. 15, no. 4, pp. 429-442, 1996.

[11] T. M. Nguyen and Q. Wu, "Gaussian-mixture-model-based spatial neighborhood relationships for pixel labeling problem," IEEE Transactions on Systems, Man, and Cybernetics, Part B: Cybernetics, vol. 42, no. 1, pp. 193-202, 2012.

[12] A. P. Dempster, N. M. Laird, and D. B. Rubin, "Maximum likelihood from incomplete data via the EM algorithm," Journal of the Royal Statistical Society. Series B (methodological), vol. 39, pp. 1-38, 1977. 
[13] J. A. Bilmes, "A gentle tutorial of the EM algorithm and its application to parameter estimation for Gaussian mixture and hidden Markov models," International Computer Science Institute, Tech. Rep., 1998.

[14] T. Denœux, "Maximum likelihood estimation from fuzzy data using the EM algorithm," Fuzzy sets and systems, vol. 183, no. 1, pp. 72-91, 2011.

[15] G. McLachlan and T. Krishnan, The EM algorithm and extensions. John Wiley \& Sons, 2007.

[16] M. A. T. Figueiredo and A. K. Jain, "Unsupervised learning of finite mixture models," IEEE Transactions on Pattern Analysis and Machine Intelligence, vol. 24, no. 3, pp. 381-396, 2002.

[17] P. Wu, Y. Liu, Y. Li, and B. Liu, "Robust prostate segmentation using intrinsic properties of TRUS images," IEEE Transactions on Medical IMAging, vol. 34, no. 6, pp. 1321-1335, 2015.

[18] R. Wagner, S. Smith, J. Sandrik, and H. Lopez, "Statistics of speckle in ultrasound B-scans," IEEE Transactions on Sonics and Ultrasonics, vol. 30, no. 3, pp. 156-163, 1983.

[19] E. Jakeman, "Speckle statistics with a small number of scatterers," Optical Engineering, vol. 23, no. 4, pp. 234 453-234 453, 1984.

[20] M. F. Insana, R. F. Wagner, D. G. Brown, and T. J. Hall, "Describing small-scale structure in random media using pulse-echo ultrasound," The Journal of the Acoustical Society of America, vol. 87, no. 1, pp. 179-192, 1990.

[21] V. Dutt and J. F. Greenleaf, "Ultrasound echo envelope analysis using a homodyned K distribution signal model," Ultrasonic Imaging, vol. 16, no. 4, pp. 265-287, 1994.

[22] R. W. Prager, A. H. Gee, G. M. Treece, and L. H. Berman, "Decompression and speckle detection for ultrasound images using the homodyned k-distribution," Pattern Recognition Letters, vol. 24, no. 4, pp. 705-713, 2003.

[23] P. M. Shankar, "Ultrasonic tissue characterization using a generalized Nakagami model," IEEE Transactions on Ultrasonics, Ferroelectrics, and Frequency Control, vol. 48, no. 6, pp. 1716-1720, 2001.

[24] V. Damerjian, O. Tankyevych, N. Souag, and E. Petit, "Speckle characterization methods in ultrasound images-A review," IRBM, vol. 35, no. 4, pp. 202-213, 2014.

[25] J. C. Seabra, F. Ciompi, O. Pujol, J. Mauri, P. Radeva, and J. Sanches, "Rayleigh mixture model for plaque characterization in intravascular ultrasound," IEEE Transactions on Biomedical Engineering, vol. 58, no. 5, pp. 1314-1324, 2011.

[26] M. Pereyra, N. Dobigeon, H. Batatia, and J. Tourneret, "Segmentation of skin lesions in 2-D and 3-D ultrasound images using a spatially coherent generalized Rayleigh mixture model," IEEE Transactions on Medical Imaging, vol. 31, no. 8, pp. 1509-1520, 2012. [Online]. Available: http://ieeexplore.ieee.org/stamp/stamp.jsp?arnumber=6168272

[27] Y. Zhang, M. Brady, and S. Smith, "Segmentation of brain MR images through a hidden Markov random field model and the expectation-maximization algorithm," IEEE Transactions on Medical Imaging, vol. 20, no. 1, pp. 45-57, 2001. 
[28] S. P. Chatzis and T. A. Varvarigou, "A fuzzy clustering approach toward hidden Markov random field models for enhanced spatially constrained image segmentation," IEEE Transactions on Fuzzy Systems, vol. 16, no. 5, pp. 1351-1361, 2008.

[29] H. Tang, J.-L. Dillenseger, X. D. Bao, and L. M. Luo, "A vectorial image soft segmentation method based on neighborhood weighted Gaussian mixture model," Computerized Medical Imaging and Graphics, vol. 33, no. 8, pp. 644-650, 2009.

[30] H. Zhang, Q. J. Wu, and T. M. Nguyen, "Incorporating mean template into finite mixture model for image segmentation," IEEE Transactions on Neural Networks and Learning Systems, vol. 24, no. 2, pp. 328-335, 2013.

[31] J.-L. Dillenseger, S. Laguitton, and E. Delabrousse, "Fast simulation of ultrasound images from a CT volume." Computers in Biology and Medicine, vol. 39, no. 2, pp. 180-186, Feb 2009.

[32] L. R. Dice, "Measures of the amount of ecologic association between species," Ecology, vol. 26, no. 3, pp. pp. 297-302, 1945.

[33] R. M. Haralick, K. Shanmugam, and I. Dinstein, "Textural features for image classification," IEEE Transactions on Systems, Man, and Cybernetics, vol. 3, no. 6, pp. 610-621, 1973.

[34] C. Li, C. Xu, C. Gui, and M. D. Fox, "Distance regularized level set evolution and its application to image segmentation," IEEE Transactions on Image Processing, vol. 19, no. 12, pp. 3243-3254, 2010.

[35] J. A. Noble and D. Boukerroui, "Ultrasound image segmentation: a survey," IEEE Transactions on Medical Imaging, pp. 987-1010, 2006. 